\title{
Arthroscopic management of neglected complex knee injury
}

\author{
Rajkumar S. Amaravathi ${ }^{1}$, Anoop Pilar ${ }^{1 *}$, Sandesh G. Manohar ${ }^{1}$, Madan Mohan Muniswamy ${ }^{1}$, \\ Fazal R. Rehman', Naveen J. Mathai ${ }^{2}$
}

\begin{abstract}
${ }^{1}$ Department of Orthopaedics, St Johns Medical College and Hospital, Bangalore, Karnataka, India
${ }^{2}$ Cardiff and Vale university Health Board, Cardiff, United Kingdom.
\end{abstract}

Received: 09 August 2020

Accepted: 15 September 2020

\section{*Correspondence:}

Dr. Anoop Pilar,

E-mail: dranoopp07@gmail.com

Copyright: ( ) the author(s), publisher and licensee Medip Academy. This is an open-access article distributed under the terms of the Creative Commons Attribution Non-Commercial License, which permits unrestricted non-commercial use, distribution, and reproduction in any medium, provided the original work is properly cited.

\begin{abstract}
Meyers and mckeevers type IV comminuted pattern of avulsion fracture of the anterior tibial eminence is not an uncommon injury, however its association with root avulsions of lateral and medial meniscus is very rare combination of injury and the management of the neglected case of this complex knee injury is challenging. A 43-year-old gentleman who came with a history of pain, recurrent instability, locking of his right knee for 2 weeks with restricted activity of daily living. He also had a road traffic accident 5 years ago. He was evaluated clinically, radiologically by X Rays and MRI Scan which revealed complete tear of anterior cruciate ligament (ACL) with loose fragments, root tear anterior horn lateral meniscus and posterior horn tear with posterior root avulsion of the medial meniscus. He was managed with arthroscopic removal of large loose body with other loose bony fragments, ACL Reconstruction with hamstring autograft, anterior root repair of lateral meniscus with pull out sutures, with all inside repair of posterior horn tear of medial meniscus using fast-fix 360 device (Smith and Nephew, Andover, MA). At 4 years follow-up the patient was analysed clinically, and the functional outcome was measured with international knee documentation committee (IKDC) and knee injury and osteoarthritis outcome score (KOOS) Scoring system which showed good outcome. Patient has returned to his pre-injury activity level with no limitation of his activity of daily living. There is no set protocol of managing these kinds of neglected complex knee injury. Sometimes you need to think out of the box. A thorough knowledge of anatomy and pathomechanics of knee combined with appropriate technique of repair to save the meniscus and reconstruction of ACL and rehabilitation can yield good result.
\end{abstract}

Keywords: Neglected avulsion, Anterior cruciate ligament, Root tear meniscus, Arthroscopic repair, Reconstruction

\section{INTRODUCTION}

A rare combination of injury involving a neglected meyers and mckeever type IV. ${ }^{1}$ comminuted fracture of tibial eminence with root avulsion of the meniscus causes increase in the peak contact pressure, failure to convert axial loads into the transverse hoop stresses. ${ }^{2}$ This leads to compromise in the load bearing ability, produce meniscal extrusion, instability, changes in biomechanics of the joint leading to accelerated degeneration of the cartilage and eventually arthritis. ${ }^{3,4}$ Non-union of the fragments will cause repeated locking of the joint mainly the extension of the knee due to the impingement of the avulsed fragment.
Reconstruction and repair of these structures will restore the contact area and peak contact pressures of the knee to near normal, which would slow down early degeneration of the joint. ${ }^{5,6}$

\section{CASE REPORT}

In this case report we describe a 43 -year-old gentleman with a high physical demand who came with a history of pain, instability, locking of his right knee for 2 weeks with restricted activity of daily living to St Johns Medical College Hospital, Bangalore. His symptoms were due to a road traffic accident sustained 5 years ago. He had 
neglected similar complaints in the past till he sought treatment with us as he could no longer carry out his activity of daily living without having great discomfort in his knee. He was evaluated clinically and radio-logically by X Rays and MRI Scan, which showed complete tear of anterior cruciate ligament (ACL) with loose bony fragments, root tear anterior horn lateral meniscus and posterior horn tear with posterior root avulsion of the medial meniscus (Figure 1). T2 weighted MRI image Showing complete tear of ACL with loose fragments, root tear anterior horn lateral meniscus and posterior horn tear with posterior root avulsion of the medial meniscus (Figure 2).

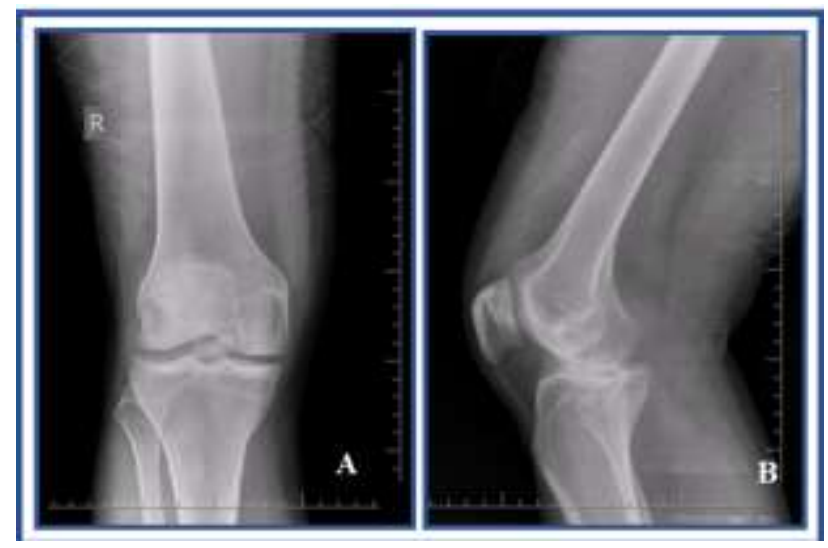

Figure 1: Showing X-ray (A) AP (B) lateral view with type IV tibial spine avulsion.

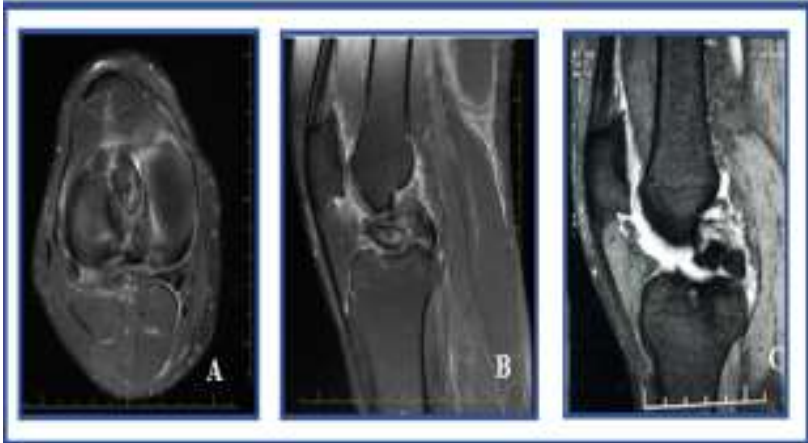

Figure 2: (A) Axial view showing bone loss at intracondylar surface of tibia (B) Coronal view

(C) Coronal view showing posterior horn tear with posterior root avulsion of the medial meniscus.

On arthroscopy it showed a comminuted avulsed fragment of the bone from the tibial eminence was sclerosed and rounded off, surrounded by fibrous tissue, and complete tear of ACL with loose fragments. Laprade et al type 4 root tear anterior horn lateral meniscus and posterior horn tear with Laprade et al type 5 posterior root avulsion of the medial meniscus were also present with grade 1 Outer bridge cartilage changes on the medial femoral condyle along with multiple loose bodies (Figure 3).
Arthroscopically loose bodies which had avulsed from the tibial eminence, the largest measuring $2-3 \mathrm{~cm}$ and multiple other loose bodies were removed through the anteromedial portal which were blocking the range of motion (ROM) (Figure 4).
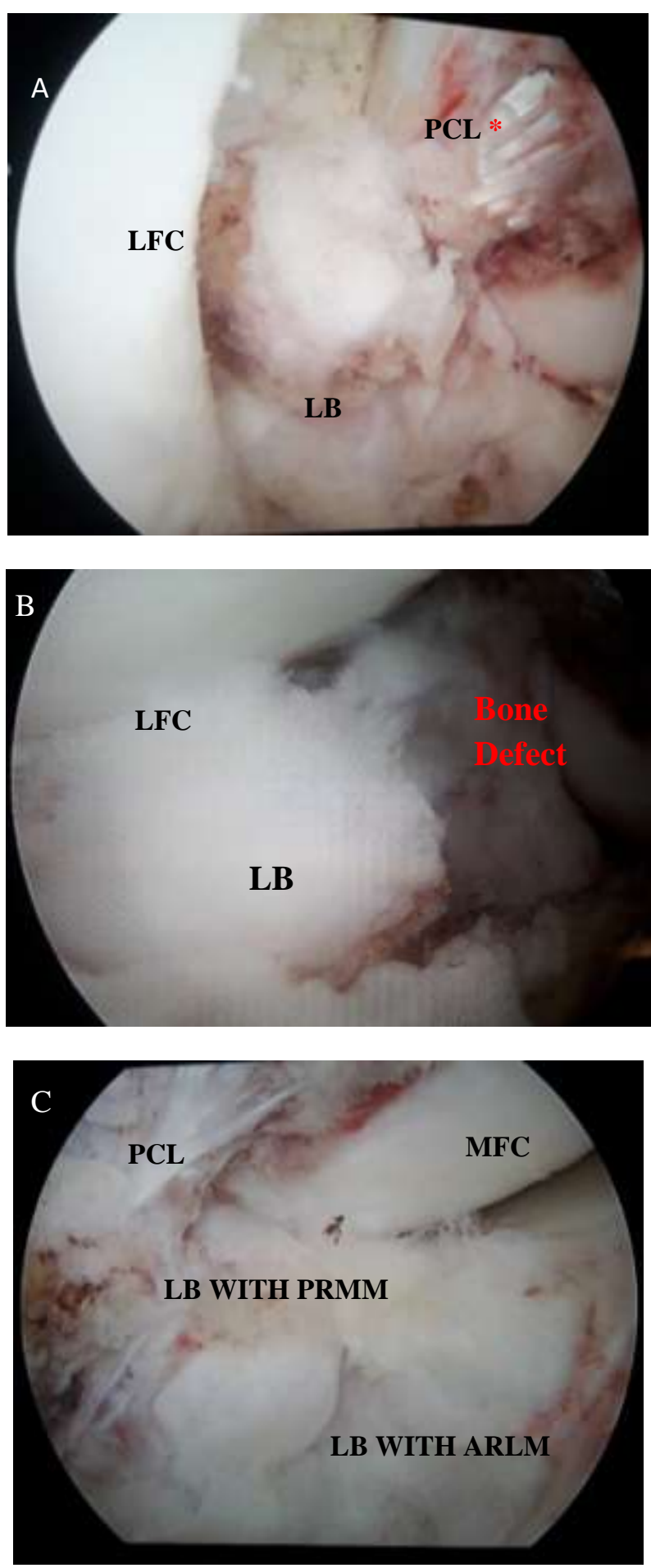

Figure 3: Arthroscopic intraoperative image showing (A) complete tear of ACL with (B) loose fragments $(\mathrm{LB})(\mathrm{C})$ root tear anterior horn lateral meniscus (ARLM) and posterior horn tear with posterior root avulsion of the medial meniscus (PRMM). 


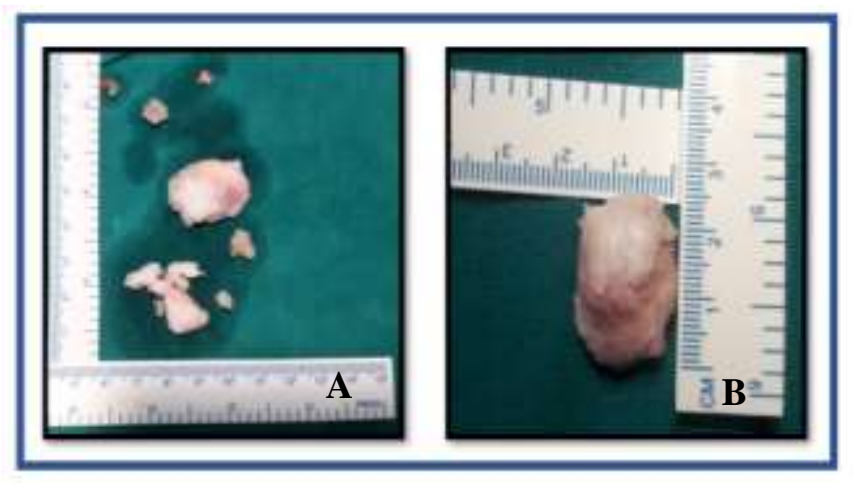

Figure 4: (A) Multiple loose fragments removed arthroscopically radially of the middle digit (B) Largest loose fragment removed arthroscopically measuring $2-3 \mathrm{~cm}$.

ACL was then reconstructed anatomically with standard arthroscopic technique using 4 tailed autologous hamstring graft. Root repair of the anterior horn of lateral meniscus was done using pull out sutures placed on the anterior horn and was retrieved through the same tibial tunnel for anterior cruciate ligament reconstruction. Ideally a separate tunnel for it is recommended, but here it would not have possible with distorted anatomy in the interspinous area of tibia to do separate tunnel for ACL and the anterior horn of lateral meniscus.

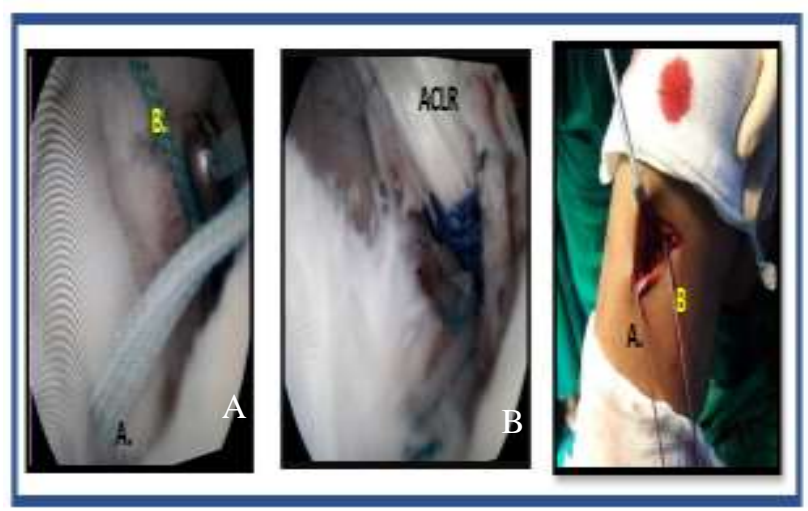

Figure 5: (A) Shuttling of the sutures of the anterior horn root tear of lateral meniscus $A$. through the tibial tunnel along with the ACL shuttle sutures B.

(B) ACL graft in situ with the sutures of the repaired anterior horn lateral meniscus. (C) Transosseous

fixation to the proximal tibia.

However, the ACL was brought anterior and anterior horn of the lateral meniscus was brought posterior through the tibial tunnel respectively and secured appropriately. The posterior horn tear of medial meniscus was repaired with all inside meniscal suture using ultra-fast-fix 360 device (Smith and Nephew, Andover, MA). Avulsed root of the posterior horn of medial meniscus was left alone, as the large bony fragment to which the root had attached was moulded nicely to the contour of the femoral and tibial condyle which was found not to limit the range of motion off knee. Secondly, removal of such a fragment would mean more damage and loss of vascularity of the meniscus. Hence a skill full neglect of medial meniscus pathology was considered (Figure 5-7).

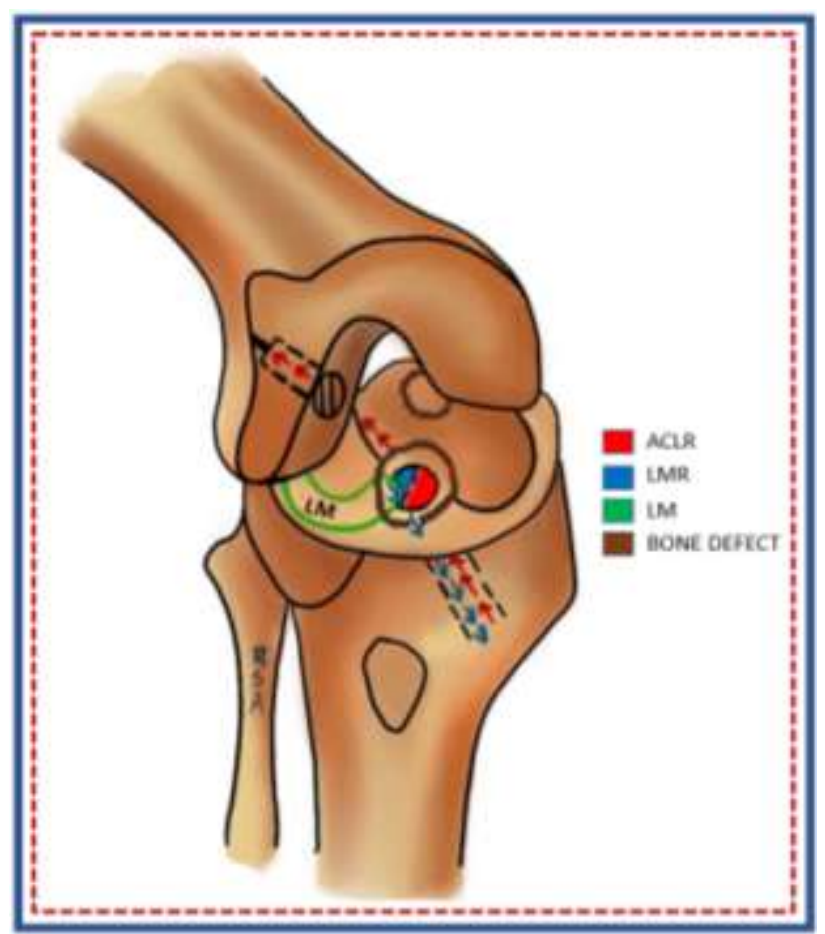

Figure 6: Graphic illustration of the placement of the tunnel on the tibia. Arrows marked in red shows tunnel for ACL. Blue arrows tunnel and shuttle sutures for anterior root LM repair.

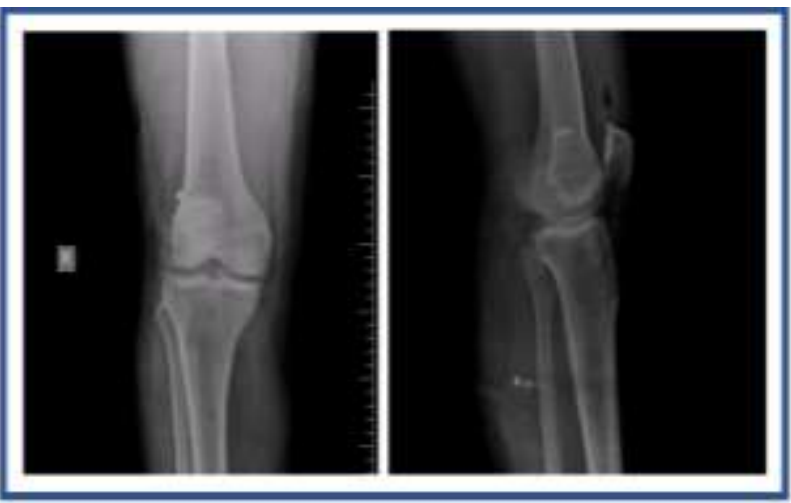

Figure 7: Showing post-operative X-ray AP \& lateral view with loose fragments removed and suspensory fixation device in situ.

Patient was kept non-weight bearing for 4 weeks with passive range of motion exercise in a safe zone of 0-60 degrees of flexion was initiated immediate post-operative day with patellar mobilization and active assisted and active quadriceps strengthening exercises. Brace was used locked at 0 degrees at night and opened 0-90 degrees in the 
day. At 4 weeks, patient was advanced in his knee flexion and weight bearing as tolerated.

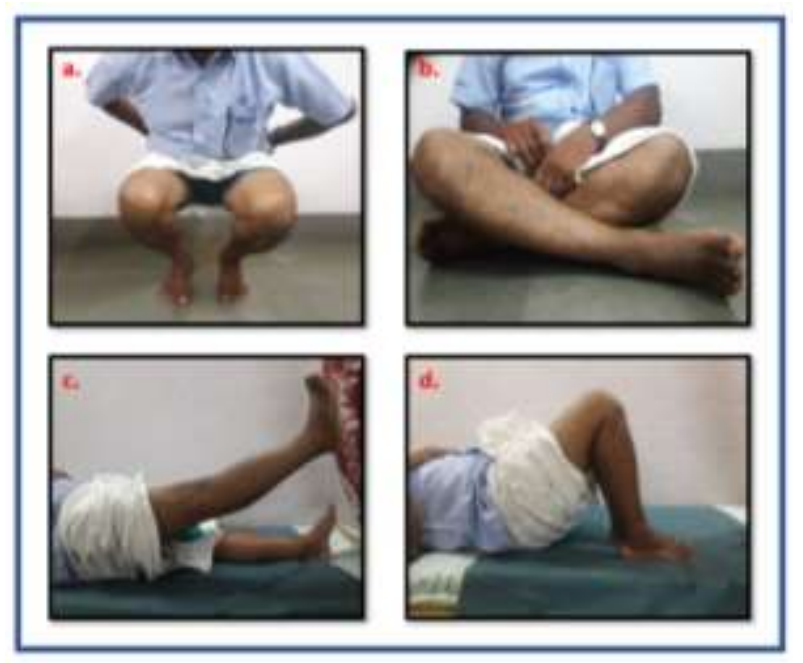

Figure 8: Showing clinical follow up images at 4 years with no disability (a) squat (b) sitting crossed leg (c) active extension with good quadriceps strength (d) complete flexion.

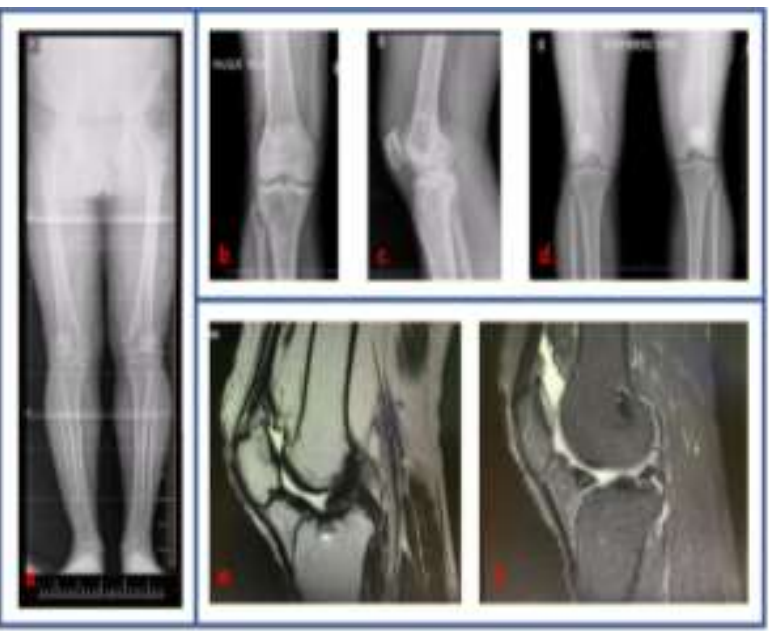

Figure 9: Showing follow up radiographs at 4 years. (a) Scanogram of both lower limbs weight bearing $\mathrm{X}$-ray-anterior posterior view showing no change in the alignment (b) Valgus stress view (c) Lateral view, (d) Rosenberg view (e, f) MRI at follow up showing healing ACL and no loss of height or contour of repair of AHLM.

Gradually the patient was eased into standard rehabilitation protocol and currently has returned to his pre-injury level of daily activity with no limitation of his activity of daily living. The post-operative IKDC, tegner $\&$ lysholm and marx activity rating score had excellent outcome in comparison to his pre-operative score (Table 1). The research analysis of the data was done using IBM SPSS Statistics data management tool version 25.0 (64-bit).
Table 1: pre and post-operative functional scores.

\begin{tabular}{|llll|}
\hline Scores & IKDC & $\begin{array}{l}\text { Tegner \& } \\
\text { Lysholm }\end{array}$ & $\begin{array}{l}\text { Marx Activity } \\
\text { Rating Scale }\end{array}$ \\
\hline $\begin{array}{l}\text { Pre- } \\
\text { Operative }\end{array}$ & $\mathbf{4 9 . 4}$ & $\mathbf{5 9}$ & $\mathbf{5 / 1 6}$ \\
\hline $\begin{array}{l}\text { Post- } \\
\text { Operative }\end{array}$ & $\mathbf{8 9 . 7}$ & $\mathbf{9 6}$ & $\mathbf{1 6 / 1 6}$ \\
\hline
\end{tabular}

\section{DISCUSSION}

Anterior Cruciate ligament is the chief stabilizer of the joint along with the meniscus, which maintains the hoop stress and internal homeostasis of the joint as the ACL deficiency along with meniscal insufficiency causes increased net force acting on the joint and causes deterioration of the cartilage causing early OA. ${ }^{7-9}$

Numerous studies have shown that regardless of the delay in the presentation, attempts at reduction should be made and have noted good outcomes in Meyers \& McKeever type. ${ }^{2,3}$ Avulsion fractures have been noted. In type 4 avulsion of tibial spine, management is controversial and most of the studies favour fragment excision and primary ACL reconstruction. ${ }^{1}$

Root avulsion of meniscus associated with ACL complete tear leads to alteration of the loading profiles of the joint compartment which results in loss of hoop stress resistance, meniscal extrusion, macro- instability of the joint which causes abnormal loading of the joint and accelerates the degenerative changes in the joint. ${ }^{10,11}$ Hence, the repair techniques in the joint and meniscal tissue preservation always gives a better clinical and functional outcome as the biomechanics of the joint is restored which improves stability, prevents meniscal extrusion, restores hoop stress and delays the progress of osteoarthritis. $^{12}$

Traditionally, meniscal root tears were treated nonoperatively or with partial meniscectomy. With numerous biomechanical studies showing the importance of the meniscal roots, there has been an increasing trend toward meniscal preservation for patients with sufficient articular cartilage, using a variety of repair strategies to restore meniscal function 3. Various methods have been described for the repair of the anterior root tear of the meniscus with advantages and disadvantages to the technique like arthroscopic all-inside techniques such as pull-out suture and suture anchor transtibial double-tunnel pull-out technique which were created at the location of the root attachment. ${ }^{13,14}$

In our case anterior root repair of lateral meniscus was done using pull out sutures which was placed on the anterior horn and retrieved through the same tibial tunnel for ACLR. Ideally a separate tunnel is advisable if the anatomy is not distorted. This was done as a separate tunnel for it would not have been possible with distorted anatomy in the interspinous area repair and the sutures were secured through trans-osseous technique in the 
proximal tibia. With this technique it can be advantageous in patients with poor bone quality in whom a suture anchor technique might not be desirable because of poor fixation and the displacement of the repaired root from its anatomical site can lead to decreased meniscal biomechanical properties with cyclic loading.

With this novel technique we used a single tunnel for the anatomical ACL Reconstruction and anatomical fixation of the anterior root of the lateral meniscus hence restoring the biomechanics of the Knee. Skillfully the root of posterior horn medial meniscus was untouched as it did not limit the natural range of movement of knee and the tibiofemoral cartilage on the medial compartment was good /near normal in spite of the non-anatomical condition of the PHMM being attached to the contoured bone fragment. As its retainment did not hinder ROM of knee and removal of the bone fragment and refixation of the root would have added more complexity to the procedure and with questionable outcome including the healing of meniscus root, a skillful negligence was deemed a right option for it. However, a PHMM root tear in normal circumstances would need a transtibial repair which would add value to the simultaneous ACLR and the outcome. A secondary trans osseous fixation at tibia would add value for the ACLR and root repair of meniscus.

At 4 years follow-up the patient was analyzed clinically, radiologically and functional outcome which was measured with IKDC and KOOS Scoring system showed good outcome. Alignment of the limb was maintained to near normal and with no evidence of progression of knee to osteoarthrosis with post-operative MRI showing healed ACL, anterior horn of lateral meniscus and posterior root of medial meniscus. Patient is satisfied with the procedure and has returned to his pre-injury activity level with no limitations of his activity of daily living (Figure 7 and 8).

\section{CONCLUSION}

There is no set protocol of managing these kinds of neglected complex knee injury. Sometimes you need to think out of the box. With principle of "do no harm" to the patient and a thorough knowledge of anatomy and pathomechanics of knee combined with appropriate technique of repair to save the meniscus and reconstruction of ACL and rehabilitation can yield good result.

Funding: No funding sources Conflict of interest: None declared

Ethical approval: Not required

\section{REFERENCES}

1. Chouhan DK, Dhillon MS, John R, Khurana A. Management of neglected ACL avulsion fractures: a case series and systematic review. Injur. 2017; 48(2):S54-60.
2. LaPrade CM, James EW, Cram TR, Feagin JA, Engebretsen L, LaPrade RF. Meniscal root tears: a classification system based on tear morphology. Am J Sports Med. 2015;43(2):363-9.

3. Bhatia S, LaPrade CM, Ellman MB, LaPrade RF. Meniscal root tears: significance, diagnosis, and treatment. Am J Sports Med. 2014;42(12):3016-30.

4. Ellman MB, LaPrade CM, Smith SD, et al. Structural Properties of the Meniscal Roots. Am J Sports Med. 2014;42(8):1881-7.

5. Tang X, Marshall B, Wang JH, et al. Lateral Meniscal Posterior Root Repair With Anterior Cruciate Ligament Reconstruction Better Restores Knee Stability. Am J Sports Med. 2019;47(1):59-65.

6. Marzo JM, Gurske-DePerio J. Effects of medial meniscus posterior horn avulsion and repair on tibiofemoral contact area and peak contact pressure with clinical implications. Am J Sports Med. 2009; 37(1):124-9.

7. Allaire R, Muriuki M, Gilbertson L, Harner CD. Biomechanical consequences of a tear of the posterior root of the medial meniscus. Similar to total meniscectomy. J Bone Joint Surg Am. 2008;90(9): 1922-31.

8. Matheny LM, Ockuly AC, Steadman JR, LaPrade RF. Posterior meniscus root tears: associated pathologies to assist as diagnostic tools. Knee Surg Sports Traumatol Arthrosc. 2015;23(10):3127-31.

9. Chung KS, Ha JK, Yeom CH, et al. Comparison of Clinical and Radiologic Results Between Partial Meniscectomy and Refixation of Medial Meniscus Posterior Root Tears: A Minimum 5-Year Follow-up. Arthroscop. 2015;31(10):1941-50.

10. Geeslin AG, Civitarese D, Turnbull TL, Dornan GJ, Fuso FA, LaPrade RF. Influence of lateral meniscal posterior root avulsions and the meniscofemoral ligaments on tibiofemoral contact mechanics. Knee Surg Sports Traumatol Arthrosc. 2016;24(5): 1469-77.

11. LaPrade CM, Jansson KS, Dornan G, Smith SD, Wijdicks CA, LaPrade RF. Altered tibiofemoral contact mechanics due to lateral meniscus posterior horn root avulsions and radial tears can be restored with in situ pull-out suture repairs. J Bone Joint Surg Am. 2014;96(6):471-9.

12. Chung KS, Ha JK, Ra HJ, Kim JG. A meta-analysis of clinical and radiographic outcomes of posterior horn medial meniscus root repairs. Knee Surg Sports Traumatol Arthrosc. 2016;24(5):1455-68.

13. Osti L, Del Buono A, Maffulli N. Anterior Medial Meniscal Root Tears: A Novel Arthroscopic All Inside Repair. Transl Med UniSa. 2014;12:41-6.

14. Menge TJ, Chahla J, Dean CS, Mitchell JJ, Moatshe G, LaPrade RF. Anterior Meniscal Root Repair Using a Transtibial Double-Tunnel Pullout Technique. Arthrosc Tech. 2016;5(3):e679-84.

Cite this article as: Amaravathi RS, Pilar A, Manohar SG, Muniswamy MM, Rehman FR, Mathai NJ.

Arthroscopic management of neglected complex knee injury. Int J Res Orthop 2020;6:1327-31. 